\title{
INFRARED BACKGROUND STARLIGHT: OBSERVATIONS AND GALAXY MODELS
}

\author{
G. G. Fazio, T. M. Dame, and S. Kent \\ Harvard-Smithsonian Center for Astrophysics \\ 60 Garden St. \\ Cambridge, MA 02138 USA
}

\begin{abstract}
The near-infrared region of the spectrum is a particularly advantageous window for observing the distribution of old, evolved stars in the galactic disk and bulge. These stars are important because they provide an excellent tracer of the overall stellar mass distribution. At shorter wavelengths extinction is a serious problem, and at longer wavelengths the flux is dominated by dust emission. A summary of the large-scale diffuse near-infrared observations of the Galaxy is presented, as is a summary of the results obtained from these data on the structure of the galactic disk and bulge. The importance of combining CO and near-infrared maps of similar resolution to determine a three-dimensional model of galactic extinction is demonstrated. The Spacelab-2 Infrared Telescope (IRT) data are used in conjunction with a proposed galactic model to make preliminary measurements of the global scale parameters of the Galaxy.
\end{abstract}

\section{INTRODUCTION}

Observations at near- and mid-infrared wavelengths of the center and the plane of our Galaxy are important since, in contrast to visible light, the galactic plane is much more transparent at infrared wavelengths.

The observed spectrum of the infrared radiation from the galactic plane is shown in Figure 1 (Giard et al. 1988), presented as the average surface brightness in the inner Galaxy $\left(8.5^{\circ}<l<35^{\circ},|b|<1^{\circ}\right)$. The spectral region from 1 to $4 \mu \mathrm{m}$ is dominated by continuum radiation from stars. Recently, emission has been detected (Giard et al. 1988) in a narrow band centered at $3.3 \mu \mathrm{m}$, which is present above the continuum emission. This feature is the characteristic signature of aromatic hydrocarbon molecules (Léger and Puget 1984). In the spectral region greater than $4 \mu \mathrm{m}$ the radiation can be explained by emission from interstellar dust grains (graphite and silicon) heated by the ambient radiation field (Cox and Mezger 1989). Most of this energy appears in the 30-300 $\mu \mathrm{m}$ spectral regions, with a broad peak at $100 \mu \mathrm{m}$. Approximately $20 \%$ of the total infrared emission is present in an additional broad peak centered at $10 \mu \mathrm{m}$. This emission is too large to be explained by the usual dust grain emission mechanisms and has been ascribed also to the emission by aromatic hydrocarbon molecules (Puget, Léger, and Boulanger 1985). In this paper we shall concentrate on observations of the continuum emission at near-infrared wavelengths, $(<4 \mu \mathrm{m})$, from stars and on what can be learned about the structure of the Galaxy from these observations.

Near-infrared radiation is emitted by relatively cool objects, $T \approx 2000 \mathrm{~K}$, with the most abundant sources in the Galaxy being late-type stars. An estimate of the percentage contribution of various spectral classes to the surface brightness of the Galaxy at K-band $(2.2 \mu \mathrm{m})$ is given by Jones et al. (1981) and Okuda (1983). The major contributors are late-type K- and M-giant stars, although early-type main sequence stars and extreme population I stars could also contribute. Late-type giant stars are part of the old, stellar population in the Galaxy, and hence knowledge of their distribution is important in the study of galactic structure and 


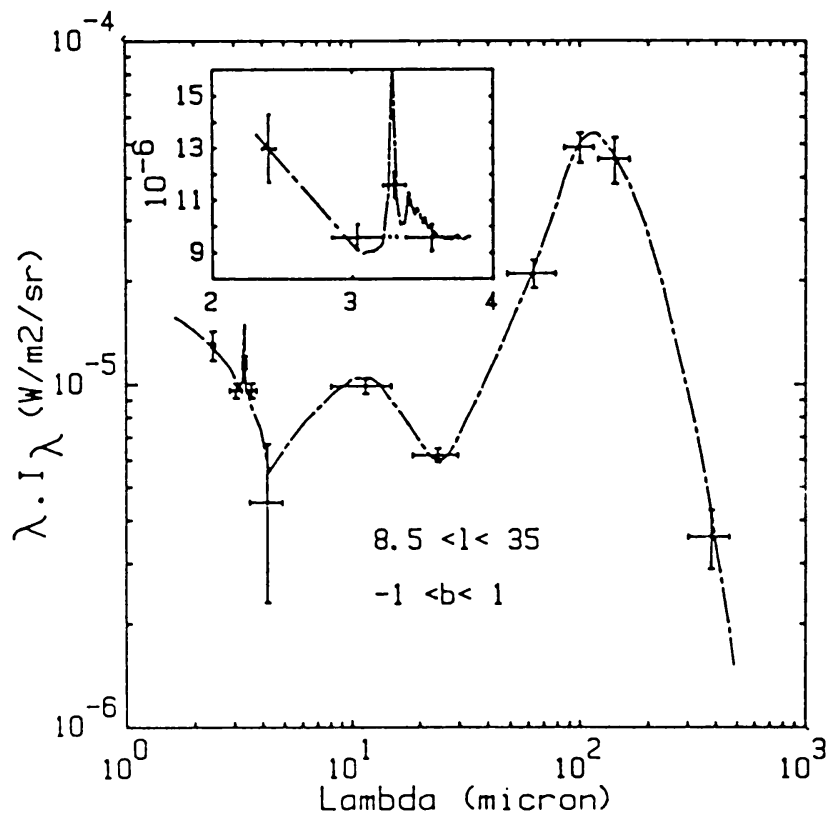

Figure 1. The averaged surface brightness $\left(\mathrm{W} / \mathrm{m}^{2} \mathrm{sr}\right)$ in the inner Galaxy $\left(8.5^{\circ}<l<35^{\circ},|b|<1^{\circ}\right)$ at infrared and submillimeter wavelengths. The insert shows the detail of the $3.3 \mu \mathrm{m}$ emission feature (Giard et al. 1988).

dynamics. At optical wavelengths, because of interstellar extinction, the study of these stars has been limited to a region within $3 \mathrm{kpc}$ of the Sun.

\section{RESULTS OF THE NEAR-INFRARED LARGE-SCALE SURVEYS}

Since the early 1970's, numerous observations have been performed to produce large-scale near-infrared maps of the galactic plane and center region. Most of these observations have been made by high-altitude balloon-borne telescopes of modest size, operating primarily in a relatively narrow wavelength band centered at $2.4 \mu \mathrm{m}$, corresponding to a spectral window in the earth's airglow emission. A limited number of observations have also been made at longer wavelengths up to $4.8 \mu \mathrm{m}$; these results have been important in determining interstellar extinction. Telescope mirror diameters have been typically $20 \mathrm{~cm}$ or less, and angular resolutions have been in the range from 0.5 to $4^{\circ}$.

Okuda (1981) has summarized the observational parameters of these large-scale photometric surveys conducted by groups at Nagoya University and the University of Melbourne (e.g. Matsumoto et al. 1982), at Kyoto University and the Institute of Space and Astronautical Science-ISAS (e.g., Oda et al. 1979), the Max-Planck-Institut für Astronomie, Heidelberg (Hoffmann, Lemke, and Frey 1978), and a combined French group (Giard et al. 1988). In addition, rocket-borne telescopes have been flown by the Air Force Geophysical Laboratories 


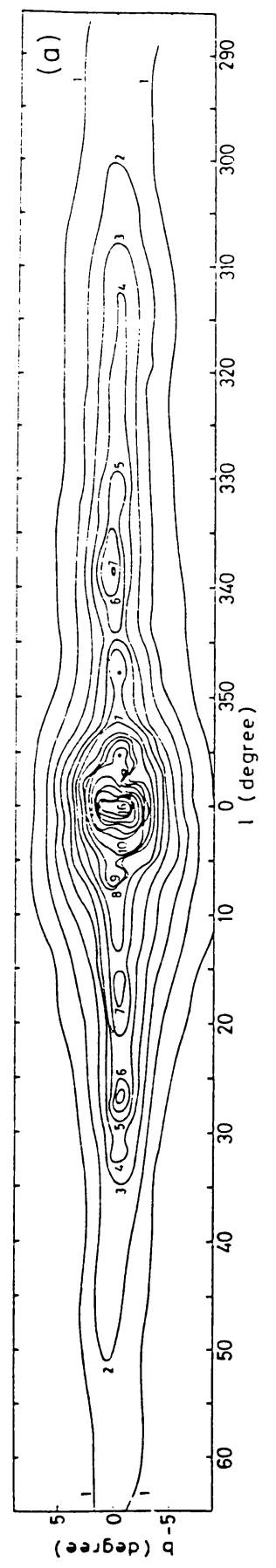

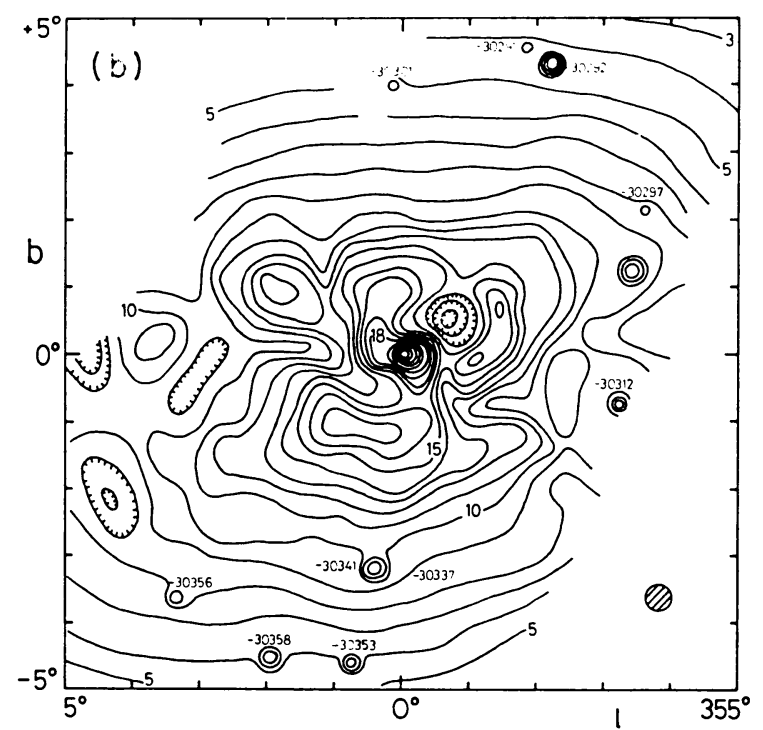

Figure 2. (a) The synthesized $2.4 \mu \mathrm{m}$ surface-brightness map of the galactic plane (Hayakawa et al. 1981). The contour lines are labelled in units of $1.0 \times 10^{-10} \mathrm{~W} /\left(\mathrm{cm}^{2} \mathrm{sr} \mu \mathrm{m}\right)$. (b) The $2.4 \mu \mathrm{m}$ surface-brightness map of the central $10^{\circ}$ of the Galaxy (Hiromoto et al. 1984). The contour lines are labelled in units of $1.0 \times 10^{-10} \mathrm{~W} /\left(\mathrm{cm}^{2} \mathrm{sr} \mu \mathrm{m}\right)$.

(AFGL; Price and Marcotte 1980) and Nagoya University/ISAS (Noguchi, Hayakawa, and Matsumoto 1981), and a 15-cm heliumcooled telescope was flown on the Spacelab-2 flight of the Space Shuttle by combined groups at the Smithsonian Astrophysical Observatory, the University of Arizona, and the NASA/Marshall Space Flight Center (Koch et al. 1988). Large-scale ground-based observations of the galactic plane using infrared source counts have been made by Kawara et al. (1982) with a multicolor photometer at I-, H-, $\mathrm{K}$-, and L-bands; $2^{\circ} \times 1^{\circ}$ maps of the galactic center region in the $\mathrm{J}$-, $\mathrm{H}-$, and K-bands have been produced by Glass, Catchpole, and White (1987); and a $30 \times 40^{\prime}$ map at K-band of the galactic center by Gatley et al. (1989).

K-band polarization measurements have been made by Okuda (1985) at $l=0^{\circ}, 20^{\circ}$, and $30^{\circ}$. The observed polarization direction is parallel to the galactic plane, and the magnitude is well correlated with interstellar extinction. The dependence of polarization on $\mathrm{H}-\mathrm{K}$ index varies with galactic longitude, and this effect is explained by assuming that the magnetic field lines are concentric with the galactic center.

The results of the balloon-borne mapping program have been summarized by Hayakawa et al. (1981) in a synthesized contour map of the $2.4 \mu \mathrm{m}$ surface brightness (Figure 2a), extending in galactic longitude from $-80^{\circ}$ to $+70^{\circ}$. Figure $2 \mathrm{~b}$ is a blowup of the central bulge of our Galaxy as observed by a balloon-borne telescope at 2.4 

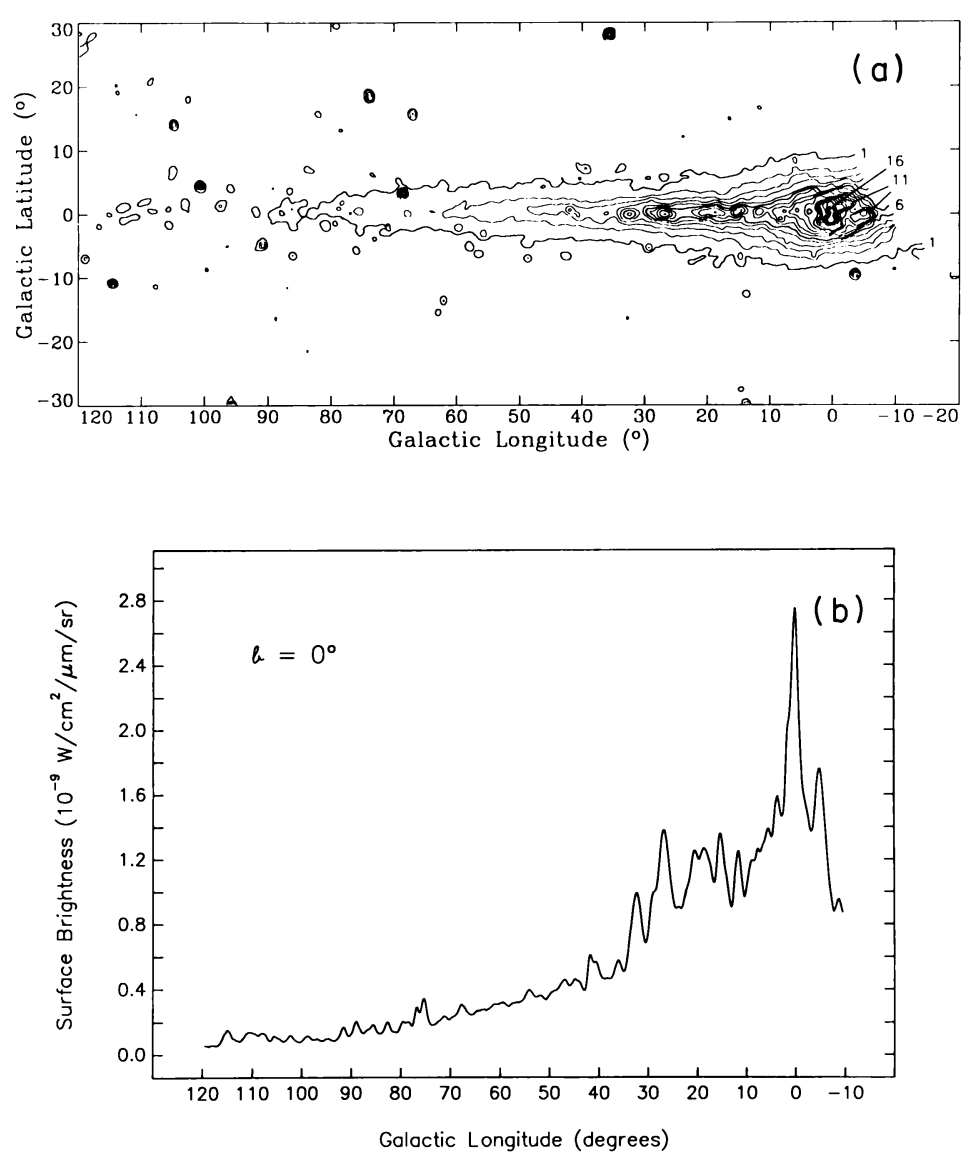

Figure 3. (a) The Spacelab-2 IRT map of the $2.4 \mu \mathrm{m}$ surface brightness of the first quadrant of the galactic plane (Koch et al. 1988). The contours are labelled in units of $1.4 \times 10^{-10} \mathrm{~W} /\left(\mathrm{cm}^{2} \mathrm{sr} \mu \mathrm{m}\right)(\mathrm{b})$ The Spacelab-2 IRT longitudinal distribution of the $2.4 \mu \mathrm{m}$ surface brightness at a galactic latitude $b=$ $0^{\circ}$ (Koch et al. 1988).

$\mu \mathrm{m}$ with a spatial resolution of $0.4^{\circ}$ (Hiromoto et al. 1984). A similar map of the bulge was also made earlier by Matsumoto et al. (1982) with an angular resolution of $0.5^{\circ}$.

The Spacelab-2 IRT maps at $2.4 \mu \mathrm{m}$ (Koch et al. 1988), which extend in galactic longitude from $-10^{\circ}$ to $+130^{\circ}$, are presented in Figures 3 and 4, and the region of the galactic bulge is shown in Figure 4b (Melnick et al. 1987). These smoothed maps, made with the N-slit star sensor in the IRT, have a resolution of about $1^{\circ}$. Compared with the balloon-borne telescope maps, the IRT data have the advantages of (a) being free from airglow contamination, (b) having a long baseline for photometric measurements, since scans were taken from the galactic plane to the pole, (c) excellent position information, (d) more uniform sensitivity calibration over the sky, and (e) measurements to galactic longitude $130^{\circ}$, which permit comparison of the luminosity distribution in the galactic center region with that in the solar neighborhood. The IRT also incorporated a cold shutter for absolute zero-point calibrations. These maps have 


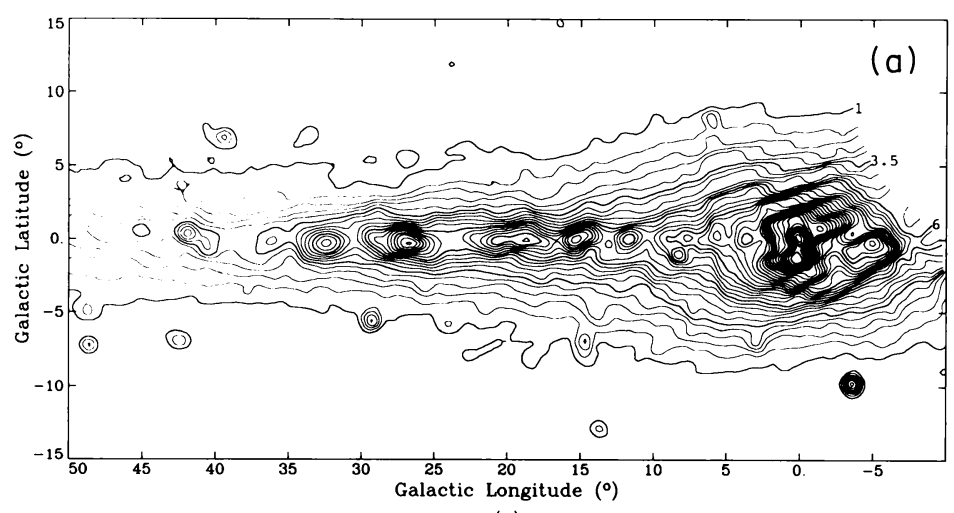

(a)

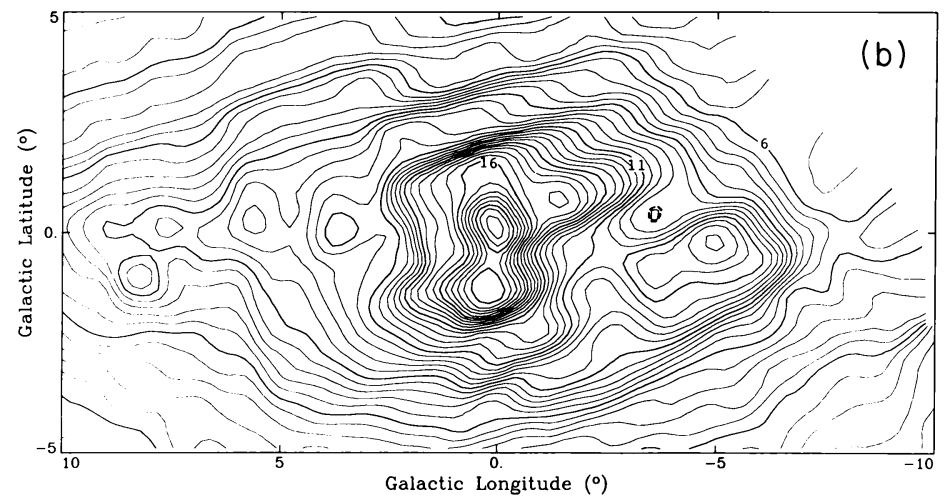

Figure 4. (a) The Spacelab-2 IRT map of the $2.4 \mu \mathrm{m}$ surface brightness of the inner disk of the Galaxy. The contours are labelled in units of $1.4 \times 10^{-10} \mathrm{~W} /\left(\mathrm{cm}^{2} \mathrm{sr} \mu \mathrm{m}\right)$. (b) The Spacelab-2 IRT map of the $2.4 \mu \mathrm{m}$ surface brightness of the central $10^{\circ}$ of the Galaxy (Melnick et al. 1987). The contours are labelled in units of $1.4 \times 10^{-10} \mathrm{~W} /\left(\mathrm{cm}^{2} \mathrm{sr} \mu \mathrm{m}\right)$.

the general appearance of an edge-on spiral galaxy. However, several important features should be noted.

\subsection{Galactic Bulge}

The "bulge" is that volume of stars in our Galaxy surrounding the galactic center, with an outer radius of about $1 \mathrm{kpc}$. Assuming a distance of $8 \mathrm{kpc}$ to the galactic center, $1 \mathrm{kpc}$ corresponds to a subtended angle of $7^{\circ}$. Frogel (1988) has summarized the optical and infrared properties of the bulge. Figures $2 \mathrm{~b}$ and $4 \mathrm{~b}$ show detailed near-infrared maps of this region. Additional near-infrared maps and analyses of the bulge have been made by Matsumoto et al. (1982); Harris, Lemke, and Hoffmann (1980); and Little and Price (1985). These maps show that the surface brightness distribution can be represented by an ellipsoid with major axis along the plane, with an axial ratio of about 0.7 . At a galactic longitude of $+10^{\circ}$, the IRT data give a latitudinal width full width at half maximum, or FWHM, for the bulge of $8^{\circ}$. The infrared 
maps also show considerable structure, which can be explained by variable interstellar extinction along the line of sight, rather than by enhanced source emission. This extinction is probably due to giant molecular clouds. Ground-based near-infrared observations have shown similar results (Glass, Catchpole, and Whitelock 1987). Matsumoto et al. (1982) have proposed that these clouds are in a molecular ring with a radius of $300 \mathrm{pc}$ around the galactic center. The infrared data can also be used to determine the bulge luminosity, mass, and mass/luminosity ratio. Previous estimates of $M / L_{\mathrm{v}}$ have ranged from 2.0 (Matsumoto et al. 1982) to 4.0 (Harris, Lemke, and Hoffmann 1980).

At the galactic center, within $\pm 0.2^{\circ}$, there exists an additional concentration of nearinfrared sources that exceeds the extrapolation of the bulge and disk stellar populations (Becklin and Neugebauer 1968; Okuda 1985; Catchpole, Glass, and Whitelock 1989; Gatley et al. 1989). Heavy, patchy extinction is also present in this region (Lebofsky 1979) and Gatley et al. (1989) have shown that the heavy extinction to the southwest of the nucleus correlates well with the circumnuclear molecular ring. A review of the stellar population in the galactic center has been given by Rieke (1989).

\subsection{Inner Thin Disk}

This component of the near-infrared radiation extends from the galactic center region to a rather sharp cutoff at galactic longitude $\pm 30^{\circ}$, having a latitudinal width (FWHM) of about $3.5^{\circ}$ as measured from the IRT data. The width would be even thinner if corrected for extinction and line-of-sight effects; some preliminary modeling of these effects shows that the corresponding exponential scale height is of the order of $150 \mathrm{pc}$. This value is much less than the 300-400 pc exponential scale height of K- and M-giant stars in the solar neighborhood. Along the plane the infrared data show a peak at galactic longitude $\sim 27^{\circ}$, tangent to the broad peak in the molecular gas density at $4 \mathrm{kpc}$. At longer wavelengths, both the IRAS data (11 and $25 \mu \mathrm{m}$ ) and the AFGL data, which measure primarily long period variable stars with very high mass-loss rates, show similar results, with an exponential scale height of $250 \mathrm{pc}$ for the inner disk (Habing 1988). Similar distributions are observed in the radio continuum, CO, farinfrared, and gamma-ray data. There are also conspicuous enhancements in the near-infrared flux in the thin disk that were once thought to correspond to tangential views of the spiral arms. But as shown later (Figures 5 and 6), these enhancements correspond to minima in the interstellar extinction, i.e., to regions between the spiral arms.

An exponential function for the radial distribution of stellar emission, fitted to the outer disk, beyond $l= \pm 30^{\circ}$, when extrapolated to the inner disk, falls well below the observed intensity. Additional sources, other than the predicted $\mathrm{K}$ - and $\mathrm{M}$-giant population, are needed. The thinness of this disk is also difficult to explain. It is not clear whether the excess emission is due to a population of young $\mathrm{M}$-supergiant stars associated with the enhanced star formation or to an overall increase in the old disk stellar density.

\subsection{Outer Disk}

The outer disk is a more extended stellar component, detectable out past $90^{\circ}$ in galactic longitude with a thickness of $5^{\circ}$ (FWHM); preliminary modeling of the outer disk shows that the corresponding exponential scale height is about 300 pc. This thickness is comparable with the scale height of $\mathrm{K}$ - and M-giant stars. The outer disk is probably the same disk observed optically in the solar neighborhood. IRAS also observed a thick, more extended disk, but with an exponential scale height of 680-1600 pc. The IRAS objects cut off suddenly at about $|l|>$ 
$90^{\circ}$, which corresponds approximately to the distance of the Sun from the galactic center (Habing 1988).

\section{ABSOLUTE CALIBRATION OF THE NEAR-INFRARED FLUX}

Frogel (1988), in his review article on the galactic bulge, suggests that a possible systematic error exists in the surface brightness $\left(\mathrm{W} / \mathrm{cm}^{2} \mathrm{sr} \mu \mathrm{m}\right)$ balloon measurements of Hiromoto et al. (1984), Figure $2 b$, because they are a factor of 2 higher than the surface brightness inferred from ground-based measurements. In the galactic bulge region, the IRT data agree in intensity with the data of Hiromoto et al. (1984). In the combined Nagoya/Kyoto balloon data (Okuda 1983; Figure 7), there exists an internal scatter of a factor of $\sim 1.5$. From $|l|=30^{\circ}$ to $40^{\circ}$, the IRT data are about a factor of 2 higher than the balloon data summarized by Hayakawa et al. (1981) and shown in Figure 2a. These differences have to be investigated further, and the IRT data will be recalibrated.

\section{COMPARISON OF NEAR-INFRARED AND CO OBSERVATIONS}

Dame et al. (1987) have mapped the galactic CO distribution with a resolution comparable with that of the IRT map (Figure 5). An important preliminary result of our comparison of these surveys, shown in Figure 6, is the strong anticorrelation of the intensities of $\mathrm{CO}$ and 2.4 $\mu \mathrm{m}$ emissions toward the inner Galaxy, the CO peaks corresponding to $2.4 \mu \mathrm{m}$ holes, and vice

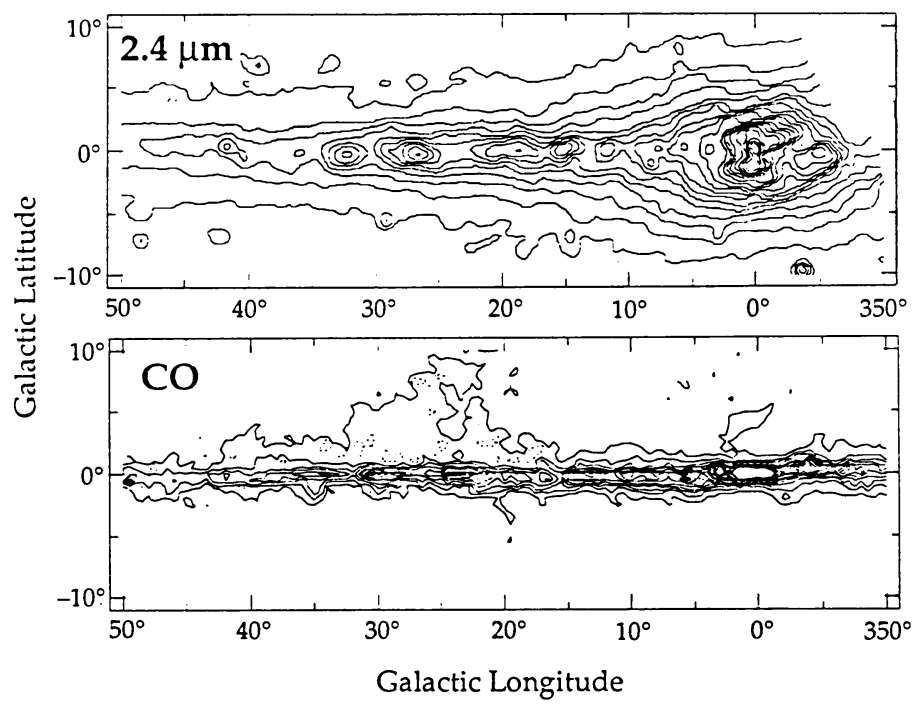

Figure 5. Comparison of the $2.4 \mu \mathrm{m}$ and CO integrated intensity maps. The $2.4 \mu \mathrm{m}$ contours range from $1.4 \times 10^{-10} \mathrm{~W} /\left(\mathrm{cm}^{2} \mathrm{sr} \mu \mathrm{m}\right)$ in unit steps. The CO data were taken from the survey of Dame et al. (1987); the contours range from 10 to $400 \mathrm{~K} \mathrm{~km} / \mathrm{s}$ in steps of $20 \mathrm{~K} \mathrm{~km} / \mathrm{s}$. 


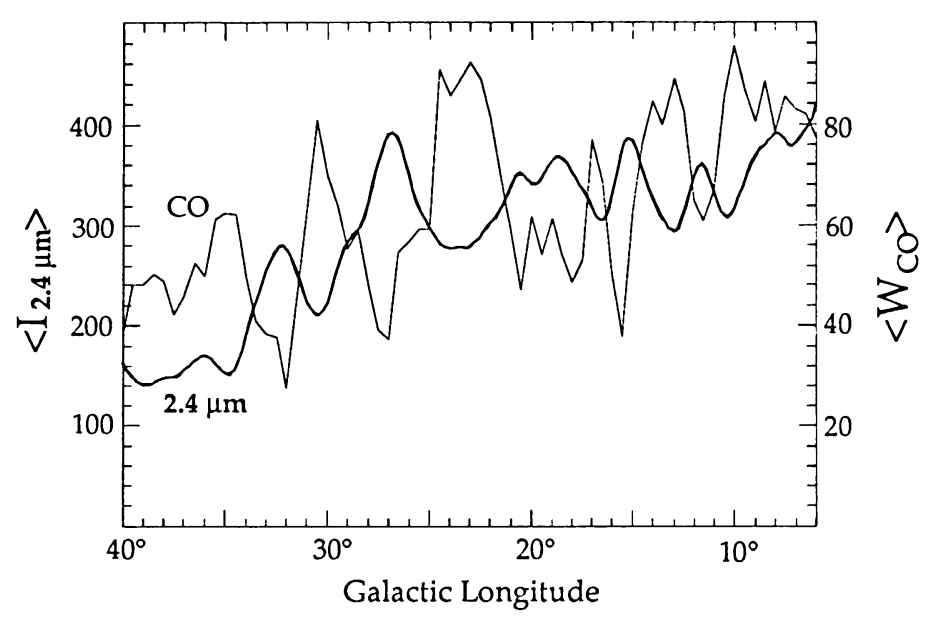

Figure 6. Comparison of the $2.4 \mu \mathrm{m}$ and CO longitude profiles. The CO intensity, from the survey of Dame et al. (1987), has been integrated over all velocities, and both curves have been averaged from $-1^{\circ}$ to $+1^{\circ}$ in galactic latitude. $W_{C O}$ is in units of $\mathrm{K} \mathrm{km} \mathrm{s}^{-1} ; \mathrm{I}_{2.4}$ is in units of $2.8 \times 10^{-12} \mathrm{~W} /\left(\mathrm{cm}^{2} \mathrm{sr}\right.$ $\mu \mathrm{m})$.

versa. Although this result has been suggested before (Okuda 1981; Harris, Lemke, and Hoffmann 1980; Glass, Catchpole, and Whitelock 1987), it has never been so dramatically exhibited over such a wide range of galactic longitude. It strongly suggests that the $2.4 \mu \mathrm{m}$ emission is coming from an old population of stars not directly associated with molecular clouds. Just as small, nearby molecular clouds are silhouetted against the optical light of the disk, in Figure 6 many giant molecular complexes in the inner spiral arms of the galaxy are evidently silhouetted against the more penetrating infrared light of the disk. Further, just as star counts provide information on the distances to very nearby molecular clouds, a detailed model of the $2.4 \mu \mathrm{m}$ emission based on $21 \mathrm{~cm}$ and $\mathrm{CO}$ surveys and the infrared light distributions discussed below (Section 5) can provide information on the distances to large atomic and molecular complexes throughout the Galaxy and so yield a rough three-dimensional map of the galactic extinction. The modeling will also yield a value for the ratio of absorption at $2.4 \mu \mathrm{m}$, $A_{2.4}$, to the $C O$ emissivity, $W_{C O}$. In a preliminary two-dimensional model of this sort, we found that the $2.4 \mu \mathrm{m}$ emission can be reasonably well fit using standard values for the ratios $N\left(H_{2}\right) / W_{C O}\left(2.3 \times 10^{20} \mathrm{~cm}^{-2} \mathrm{~K}^{-1} \mathrm{~km}^{-1} \mathrm{~s}\right.$; Strong et al. 1988$), \mathrm{N}(\mathrm{H}) / \mathrm{A}_{v}\left(1.9 \times 10^{21} \mathrm{~cm}^{-2} \mathrm{mag}\right.$; Bohlin, Savage, and Drake 1978), and $A_{2.4} / A_{v}$ (13.6; van de Hulst 1957). Future threedimensional models with more sophisticated infrared light distributions should provide useful constraints on these ratios.

\section{GALACTIC MODELS}

For edge-on spiral galaxies, the spatial distribution of the infrared surface brightness in cylindrical coordinates, $v(R, z)$, can be represented by the equation:

$$
v(R, z)=v_{o} \exp (-R / h) \operatorname{sech}^{2}\left(z / z_{o}\right) \text {. }
$$


For $z / z_{o} \gg 1$ :

$$
\operatorname{sech}^{2}\left(z / z_{o}\right) \approx 4 \exp \left(-2 z / z_{o}\right) ; \text { and } z_{\text {exp }}=z_{o} / 2,
$$

(van der Kruit and Searle 1981), with three adjustable constants $\left(\mathrm{v}_{o}, \mathrm{~h}\right.$, and $\left.z_{o}\right)$, where $\mathrm{h}$ and $z_{o}$ are the scale lengths in the radial direction and normal to the plane, respectively.

For the galactic bulge light distribution, a reasonable approximation is that the projected light distribution or surface brightness, $\mu$, follows the equation:

$$
\begin{gathered}
\mu=\mu_{o} \exp \left(-s / h_{B}\right), \\
\text { where } s=\left[R^{2}+z^{2} /(1-\varepsilon)^{2}\right]^{1 / 2},
\end{gathered}
$$

with $\varepsilon$ the ellipticity of the spheroidal bulge, and $\mu_{o}, h_{B}$, and $\varepsilon$ adjustable parameters.

From the results of the balloon-borne large-scale near-infrared surveys, various authors have inferred the stellar mass distribution of the inner galaxy. A summary of early models and of the interpretation of the large-scale emission from the galactic plane has been presented by Drapatz (1981).

Okuda (1983; 1985), by combining balloon-borne and ground-based near-infrared observations, constructed a comprehensive model of the inner Galaxy based on a bulge-plus-disk contribution. To explain the large flux near the galactic center (i.e., within $\sim 20 \mathrm{pc}$ ), an additional component had to be added with a population of very luminous $\mathbf{M}$-giant or M-supergiant stars about 100 times higher than the fraction found in the solar neighborhood. Evidence for an overpopulation of luminous giants in the central $20 \mathrm{pc}$ of the Galaxy has also been found by Lebofsky and Rieke (1987). To explain the peak in the longitudinal distribution of the surface brightness observed at $l=27^{\circ}$, an appreciable concentration of very luminous M-giants or Msupergiants was proposed in an annulus located at $4 \mathrm{kpc}$ from the galactic center. Nearinfrared star counts in this region seem to confirm this assumption. In Okuda's model the excess can be explained only by assuming a luminous M-giant overpopulation by a factor of 20 compared with the average population in the solar neighborhood. A problem with these assumptions is that the expected excess of early-type O- and B-supergiants is not present. A summary of Okuda's model is shown in Figure 7.

Oda (1985) modeled the distribution of stellar radiation in the $4 \mathrm{kpc}$ ring assuming that the $2.4 \mu \mathrm{m}$ volume emissivity is dominated by M-giant and M-supergiant stars with $80 \%$ of the emissivity due to M-giants heavier than 2 to $3 \mathrm{M}_{\odot}$, i.e., evolved late B or early A stars. This theory incorporates a bimodal star formation model, proposed by Güsten and Mezger (1982), in which only medium- and high-mass stars form in spiral arms and low-mass stars form in the interarm regions. All stars heavier than a few solar mass are assumed to have the same spatial distribution and hence a scale height distribution and a number density ratio similar to that of OB stars.

Estimates of some of the scale parameters in the above equations for our Galaxy have given amazingly disparate results. For example, the estimates of the radial exponential scale length, $\mathrm{h}$, of the disk range from $2.2 \mathrm{kpc}$ (Jones et al. 1981) and $2.3 \mathrm{kpc}$ (Okuda 1983) to 5.5 kpc (van der Kruit 1986). At least three reasons for such discrepant results can be identified. First, the disk is not perfectly exponential, and the different estimates refer to different parts of the Galaxy: the 2.2 and $2.3 \mathrm{kpc}$ estimates refer approximately to $\mathrm{R}<5 \mathrm{kpc}$, while the $5.5 \mathrm{kpc}$ estimate refers to the solar neighborhood $(\mathrm{R} \approx 8 \mathrm{kpc})$. There are notable deviations from the exponential law in the near-infrared emission at $l=30^{\circ}$, corresponding to the $4 \mathrm{kpc}$ ring of molecular gas. Second, there have been variations in the absolute near-infrared flux measurements by various groups. Third, corrections for interstellar absorption have not properly allowed for the fact that the dust distribution is clumpy (effectively reducing the total opacity 


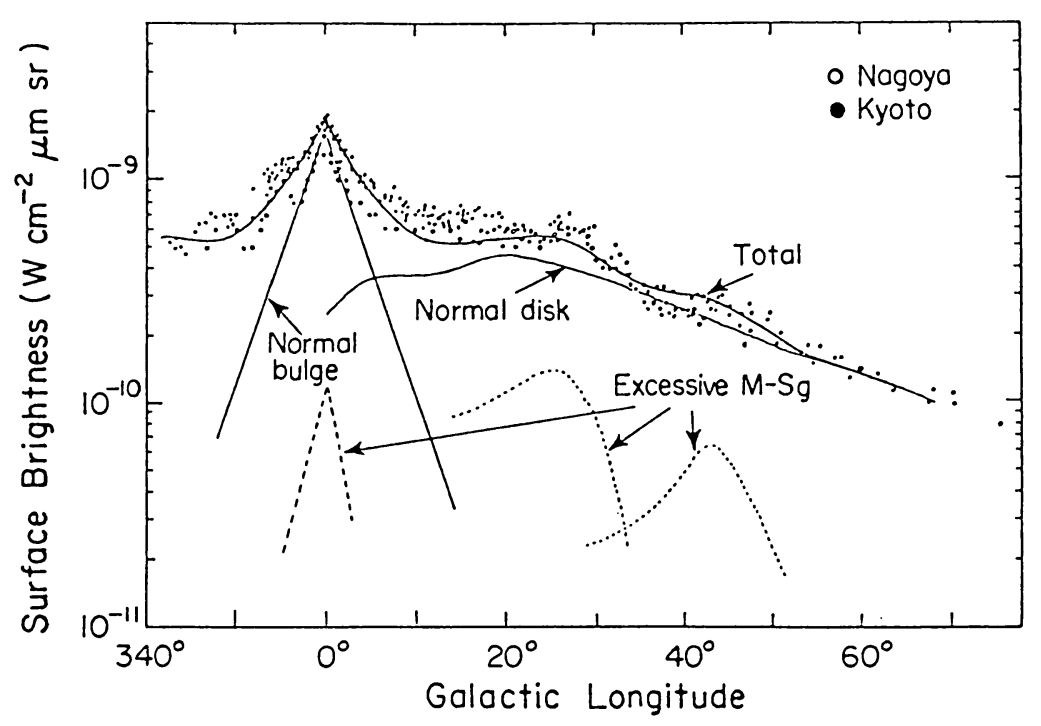

Figure 7. Model of $2.4 \mu \mathrm{m}$ surface brightness distribution calculated by Okuda (1983) compared with the data from the Nagoya and Kyoto balloon-borne telescope experiments.

compared with an assumption of uniform distribution); none of the models have included such a correction.

Using the Spacelab-2 IRT data, a more accurate measure of the global scale parameters should be possible, and a new model for the three-dimensional light distribution can be derived. Using the IRT data and CO maps in tandem, a more accurate three-dimensional map of galactic extinction for $l>20^{\circ}$ can be derived, which can then be incorporated into the model. The model will also incorporate the following features: deviations in the radial disk profile from exponential; variations in the vertical scale height, $z_{o}$, with radius; deviations of the vertical distribution of light from a $\operatorname{sech}^{2}$ law; and geometrical considerations due to our position in the Galaxy.

Using the IRT data to fit the adjustable parameters, preliminary calculations (using a simple dust extinction model) have given the following results (Figure 8). For the outer galactic disk the following parameters for equations (1) and (2) have been determined:

$$
\mathrm{h}=4 \mathrm{kpc} ; z_{o}=600 \mathrm{pc} ;\left(z_{\exp }=300 \mathrm{pc}\right) ; \mu_{o}=177 L \rho \mathrm{pc}^{2},
$$

where $\mu_{o}$ is the V-band surface brightness, assuming a value $\mathrm{V}-\mathrm{K}=3.2$. In the solar neighborhood, the V-band surface brightness was determined to be $24 \mathrm{~L}_{\odot} / \mathrm{pc}^{2}$, in agreement with direct star counts (Bachall 1984). Using $M / L_{v}=3$, the total disk luminosity is $1.8 \times 10^{10} \mathrm{~L}_{\odot}$ and the total disk mass is $5.3 \times 10^{10} \mathrm{M}_{\odot}$.

For the galactic bulge, assuming $\varepsilon \approx 0.35$, the parameters are:

$$
\mathrm{h}_{B}=540 \mathrm{pc} ; \mu_{o}=4200 L \delta \mathrm{pc}^{2},
$$

giving a total bulge luminosity of $4 \times 10^{9} \mathrm{~L} \odot$. Assuming a projected stellar velocity dispersion of $122 \mathrm{~km} / \mathrm{s}$ at $\mathrm{z}=600 \mathrm{pc}$, the value of $\mathrm{M} / \mathrm{L}_{\mathrm{v}}$ derived is 4.6 . 

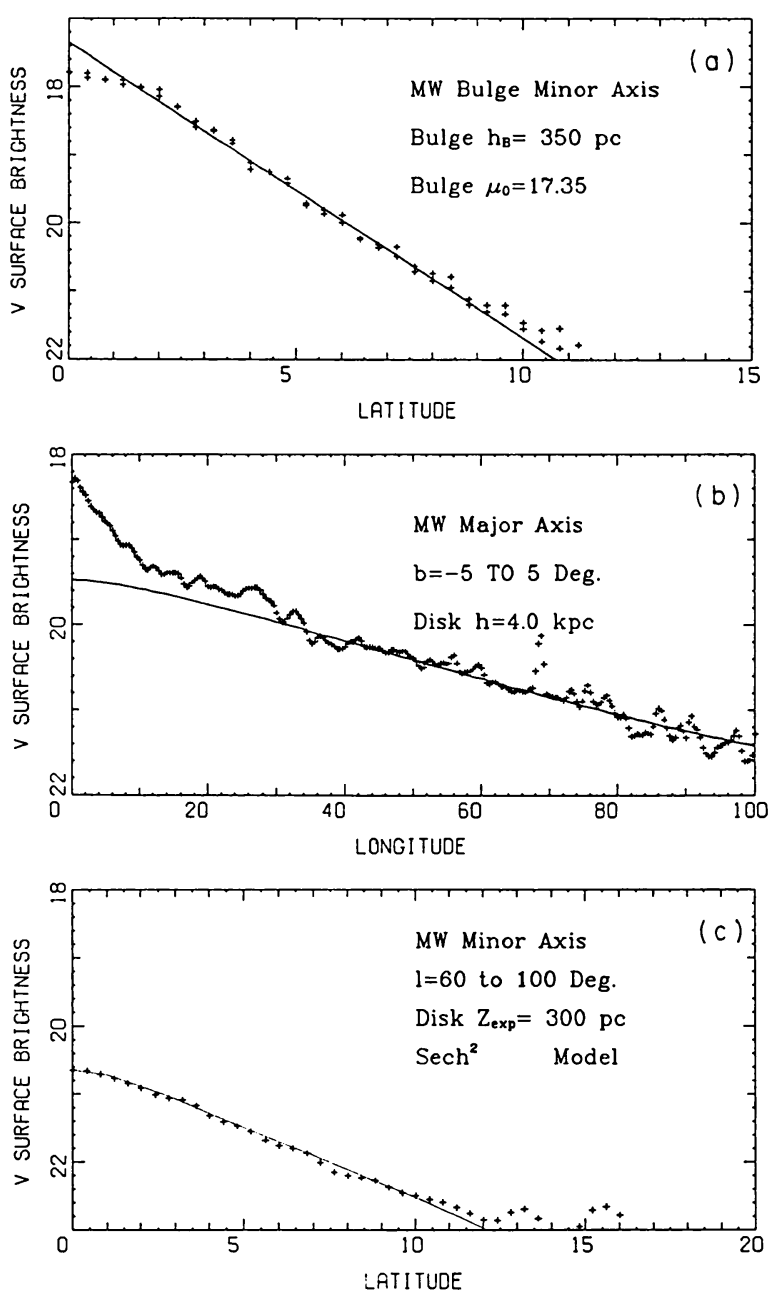

Figure 8. Comparison of the model and observed $2.4 \mu \mathrm{m}$ data from Spacelab-2 for (a) the galactic bulge minor axis; (b) the galactic disk major axis (R); and (c) the galactic disk minor axis (z). The ordinate is the V-band intensity in units of mag/arcsec ${ }^{2}$.

This model however, does not incorporate the enhanced emission in the galactic center or the excess emission in the inner disk.

\section{FUTURE PROGRAMS}

When a more careful and detailed analysis of the Spacelab-2 IRT data is carried out, we expect to be able to derive a number of useful results. These include: 
(a) distribution of dust in galactic disk;

(b) a value for the $2.4 \mu \mathrm{m}$ absorption-to- $\mathrm{CO}$ emissivity ratio, $\mathrm{A}_{2.4} / \mathrm{W}_{C O}$, which will constrain the more important ratios $\mathrm{N}\left(\mathrm{H}_{2}\right) / \mathrm{W}_{C O}$ and $\mathrm{A}_{2.4} / \mathrm{A}_{v}$;

(c) structural parameters of the Galaxy;

(d) comparison of the galactic luminosity distribution with the mass distribution;

(e) comparison of near-infrared emission with long-wavelength IRAS emission to determine if the emission peak at $l=30^{\circ}$ is due to a population of young supergiants associated with enhanced star formation or to an overall increase in the old disk stellar density;

(f) a better understanding of bulge dynamics; and

(g) comparison of the near-infrared luminosity profiles of our Galaxy with other galaxies. As an auxiliary part of the program, ground-based optical (S. Kent) and infrared images (G. Rieke) of external galaxies similar to our Galaxy have been obtained in order to find which galaxies have bulge and disk luminosity profiles most like those of the Milky Way, and to determine, among other things, the range of variability of bulge luminosity profiles.

\section{REFERENCES}

Bachall, J. N. 1984, Astrophys. J., 276, 169.

Becklin, E. E., and Neugebauer, G. 1968, Astrophys. J., 151, 145.

Bohlin, R. C., Savage, B. D., and Drake, J. F. 1978, Astrophys. J., 224, 132.

Catchpole, R. M., Glass, I. S., and Whitelock, P. 1989, IAU Symposium 136, The Center of the Galaxy, ed. M. Morris (Dordrecht: Kluwer Academic Publishers), p. 75.

Cox, P., and Mezger, P.G. 1989, Astron. Astrophys. Rev., 1, 49.

Dame, T. M., Ungerechts, H., Cohen, R. S., de Geus, E. J., Grenier, I. A., May, J., Murphy, D. C., Nyman, L- $\AA$, and Thaddeus, P. 1987, Astrophys. J., 322, 706.

Drapatz, S. 1981, in IAU Symposium 96, Infrared Astronomy, ed. C. G. Wynn-Williams and D. P. Cruikshank (Dordrecht: D. Reidel), p. 261.

Frogel, J. A. 1988, Ann. Rev. Astron. Astrophys., 26, 51.

Gatley, I., Joyce, R., Fowler, A., DePoy, D. and Probst, R. 1989, in IAU Symposium 136, The Center of the Galaxy, ed. M. Morris (Dordrecht: Kluwer Academic Publishers), p. 361.

Giard, M., Pajot, F., Lamarre, J. M., Serra, G., Caux, E., Gispert, R., Léger, A., and Rouan, D. 1988, Astron. Astrophys., 201, L1.

Glass, I. S., Catchpole, R. M., and Whitelock, P. A. 1987, M. N. R. A. S., 227, 373.

Güsten, R., and Mezger, P. G. 1982, Vistas Astron., 26, 159.

Habing, H. J. 1988, Astron. Astrophys., 200, 40.

Harris, A. W., Lemke, D., and Hoffmann, W. 1980, Astrophys. Space Science, 72, 111.

Hayakawa, S., Matsumoto, T., Murakami, H., Uyama, K., Thomas, J. A., and Yamagami, T. 1981, Astron. Astrophys., 100, 116.

Hoffmann, W., Lemke, D., and Frey, A. 1978, Astron. Astrophys., 70, 427.

Hiromoto, N., Maihara, T., Mizutani, K., Takami, H., Shibai, H., and Okuda, H. 1984, Astron. Astrophys., 139, 309.

Jones, T. J., Ashley, M., Hyland, A. R., and Ruelas-Mayorga, A. 1981, M.N.R.A.S., 197, 413.

Kawara, K., Kozasa, T., Sato, S., Kobayashi, Y., and Okuda, H. 1982, Publ. Aston. Soc. Japan, 34, 389.

Koch, D. G., Melnick, G. J., Fazio, G. G., Rieke, G. H., Low, F. J., Hoffmann, W., Young, E. T., Urban, E. W., Simpson, J. P., Witteborn, F. C., Gautier, III, T. N., and Poteet, W. 1988, Astrophys. Lett. and Comm., 27, 211.

Lebofsky, M. 1979, Astron. J., 84, 324.

Lebofsky, M. J., and Rieke, G. H. 1987, in American Institute of Physics Proc. 155, The Galactic Center, ed. D. C. Baker (New York: AIP), p. 79.

Léger, A., and Puget, J.L. 1984, Astron. Astrophys., 137, L5.

Little, S. J., and Price, S. D. 1985, Astronom. J., 90, 1812. 
Matsumoto, T., Hayakawa, S., Koizumi, H., Murakami, H., Uyama, K., Yamagami, T., and Thomas, J. A. 1982, in American Institute of Physics Conference Proceedings 83, The Galactic Center, ed. G. R. Riegler and R. D. Blandford (New York: AIP), p. 48.

Melnick, G. J., Fazio, G. G., Koch, D. G., Rieke, G. H., Young, E. T., Low, F. J., Hoffmann, W. F., and Gautier, T. N. 1987, in American Institute of Physics Proc. 155, The Galactic Center, ed. D. C. Baker (New York: AIP), p. 157.

Noguchi, K., Hayakawa, S., and Matsumoto, T. 1981, Publ. Astron. Soc. Japan, 33, 583.

Oda, N., Maihara, T., Sugiyama, T., and Okuda, H. 1979, Astron. Astrophys., 72, 309.

Oda, N. 1985, Astron. Astrophys., 145, 45.

Okuda, H. 1981, in IAU Symposium 96, Infrared Astronomy, ed. C. G. Wynn-Williams and D. P. Cruikshank (Dordrecht: D. Reidel), p 247.

Okuda, H. 1983, preprint.

Okuda, H. 1985, in IAU Symposium 106, The Milky Way Galaxy, ed. H. van Woerden, R. J. Allen, and W. B. Burton (Dordrecht: D. Reidel), p. 123.

Price, S. D., and Marcotte, L. P. 1980, AFGL Technical Report, AFGL-TR-80-0182 (AD-A0100-289).

Puget, J. L., Léger, A., and Boulanger, F. 1985, Astron. Astrophys., 142, L19.

Rieke, G. H. 1989, in IAU Symposium 136, The Center of the Galaxy, ed. M. Morris (Dordrecht: Kluwer Academic Publishers), p. 21.

Strong, A. W., Bloemen, J. B. G. M., Dame, T. M., Grenier, I. A., Hermsen, W., Lebrun, F., Nyman, L.-A, Pollock, A. M. T., and Thaddeus, P. 1988, Astron. Astrophys., 207, 1.

van de Hulst, H. C. 1957, Light Scattering by Small Particles (New York: Wiley, Chapman, and Hall).

van der Kruit, P. C., and Searle, L. 1981, Astron. Astrophys., 95, 105.

van der Kruit, P. C. 1986, Astron. Astrophys., 157, 230.

M. Longair: If you make the simplest corrections for interstellar extinction in the $2.4 \mu \mathrm{m}$ map of the galaxy, do you obtain a very smooth stellar distribution as appears to be the case in infrared camera images of external galaxies?

G. Fazio: Yes, except that additional sources must be added to the smooth distribution in the $5 \mathrm{kpc}$ molecular ring and at the galactic center.

H. Okuda: In addition to the surface photometry of the Galaxy in the near infrared, we have made star counts at the $\mathrm{H}$ - and $\mathrm{K}$-bands in selected regions of the galactic plane. The number density of the sources shows a similar behaviour as seen in the surface photometry. The intrinsic luminosity of the sources is compatible with that of supergiants or bright giants, if corrected for interstellar extinction. In order to explain the results, we also have to introduce an excess of supergiants or bright giant stars in the galactic central region and the $5 \mathrm{kpc}$ region. 